\title{
骨架结构对大环主体化合物作为阴离子受体性能的影响
}

\author{
魏金燕 ${ }^{a}$ 徐立进 $*, a$ 龚汉元 $*, b$ \\ ( ${ }^{a}$ 中国人民大学化学系 北京 100872) \\ $\left({ }^{b}\right.$ 北京师范大学化学学院 北京 100875$)$
}

\begin{abstract}
摘要 通过文献报道的方法，高效合成了大环化合物环 [2](2,6-二(1H-咪唑基)吡啶[2](1,4-二亚甲基苯)(1 $\left.{ }^{4+}\right)$ 和环[2](2,6二 $\left(1 H\right.$-咪唑基)吡啶[2](1,2-二亚甲基苯) $\left(2^{4+}\right)$. 通过溶液中核磁 ${ }^{1} \mathrm{H}$ 谱、气相电喷雾电离质谱(ESI-MS)及固相单晶衍射方 法, 详细考察了这两个大环主体化合物与一系列体积较小、形状各异的无机阴离子客体间的相互作用. Job's plot 研究结 果表明与 $\mathbf{2}^{4+}$ 相比, 大环主体 $\mathbf{1}^{4+}$ 能够结合等量或者更多的阴离子客体; 结合常数的计算表明, 对于易于形成分子间氢 键与大环主体进行复合的阴离子即 $\mathrm{Cl}^{-}, \mathrm{N}_{3}^{-}, \mathrm{NO}_{3}^{-}$或 $\mathrm{HSO}_{4}^{-}, 2^{4+}$ 与该类阴离子进行 $1: 1$ 复合的结合常数 $\left(K_{\mathrm{a} 1}\right)$ 总是大 于甚至是远大于 $\mathbf{1}^{4+}$. 但是对于较难形成分子间氢键，随着离子半径的增大导致极化性增强，更易于发生 anion- $\pi$ 作用 的离子如 $\mathrm{Br}^{-}$和 $\mathrm{I}^{-}, \mathbf{1}^{4+}$ 与它们的结合常数近于甚至大于 $\mathbf{2}^{4+}$. 推测产生上述现象的原因是由于 $\mathbf{2}^{4+}$ 具有紧凑的骨架结构, 使四个酸性较强的咪唑盐基 2 位 $\mathrm{C}-\mathrm{H}$ 位点能够有效协同，与体积较小的阴离子同时形成强的分子间氢键; 而 $\mathbf{1}^{4+}$ 的骨 架结构使得上述位点的空间距离较大，具有咪唑盐基团 2 位 $\mathrm{C}-\mathrm{H}$ 键难以全部参与对阴离子的相互作用，而更易于同时 与更多的阴离子结合，并更易于发生 anion- $\pi$ 的协同作用. 上述结果展示了大环主体化合物的骨架结构将控制其空腔的 大小、形状及与客体阴离子产生分子间氢键相互作用的 $\mathrm{C}-\mathrm{H}$ 键位点的空间分布, 从而极大地影响主客体之间复合的 模式(如化学计量比和结合常数等).
\end{abstract}

关键词 大环化合物; 骨架结构; 弱相互作用; 阴离子复合

\section{Backbone Effect of Macrocycle Host Compound as Anion Receptor}

\author{
Wei, Jinyan ${ }^{a} \quad \mathrm{Xu}, \mathrm{Lijin}^{*, a} \quad$ Gong, Hanyuan ${ }^{*, b}$ \\ $\left({ }^{a}\right.$ Department of Chemistry, Renmin University of China, Beijing 100872) \\ $\left({ }^{b}\right.$ College of Chemistry, Beijing Normal University, Beijing 100875)
}

\begin{abstract}
Two novel macrocycle hosts with flexible frameworks and cavities, cyclo[2](2,6-di(1H-imidazol-1-yl)pyridine)[2] (1,4-dimethylenebenzene) $\left(\mathbf{1}^{4+}\right)$ and cyclo[2](2,6-di(1H-imidazol-1-yl)pyridine)[2](1,2-dimethylenebenzene) $\left(\mathbf{2}^{4+}\right)$, were synthesized with high yields via cyclization reactions between 2,6-di(1H-imidazol-1-yl)pyridine and 1,4-bisbromomethyl benzene or 1,2-bisbromomethyl-benzene. Herein, the interactions between $\mathbf{1}^{4+}$ or $\mathbf{2}^{4+}$ and a series of inorganic anionic guests were studied in detail via the following methods: (1) ${ }^{1} \mathrm{H}$ NMR spectroscopy in $d_{6}$-DMSO solution; (2) electrospray ionization mass spectrometry (ESI-MS) in gas phase; (3) single crystal X-ray crystallography in solid state. It is noted that the anionic guest species has different shapes, namely anions with ball shapes like $\mathrm{Cl}^{-}, \mathrm{Br}^{-}, \mathrm{I}^{-}$; linear anion $\mathrm{N}_{3}^{-}$; triangle $\mathrm{NO}_{3}^{-}$or tetrahedron $\mathrm{HSO}_{4}{ }^{-}$. The study found that $\mathbf{1}^{4^{+}}$can bind more small inorganic anion species than macrocycle $\mathbf{2}^{4+}$. In addition, the result implied that when the inorganic anion guest acts as strong intermolecular hydrogen bond acceptor (i.e. anionic guest $\left(\mathrm{A}^{-}\right)$such as $\mathrm{Cl}^{-}, \mathrm{N}_{3}^{-}, \mathrm{NO}_{3}^{-}$or $\left.\mathrm{HSO}_{4}^{-}\right)$, the associate constant $\left(K_{\mathrm{a}}\right)$ maintains $K_{\mathrm{a}}\left[2^{4+} \cdot \mathrm{A}^{-}\right]^{3+}>K_{\mathrm{a}}\left[\mathbf{1}^{4+} \cdot \mathrm{A}^{-}\right]^{3+}$; on the contrary, when anionic guest $\mathrm{A}^{-}\left(\mathrm{Br}^{-}\right.$or $\left.\mathrm{I}^{-}\right)$is hard to form intermolecular hydrogen bonds but easy contribute to anion- $\pi$ interaction, the association constants of the $1: 1$ (host:guest) complexes follow another trend (i.e. $K_{\mathrm{a}}\left[\mathbf{2}^{4+} \cdot \mathrm{A}^{-}\right]^{3+}<K_{\mathrm{a}}\left[\mathbf{1}^{4+} \cdot \mathrm{A}^{-}\right]^{3+}$ when $\mathrm{A}^{-}$is $\mathrm{Br}^{-}$or $\mathrm{I}^{-}$). It is suggested that the skeleton of macrocycle host $2^{4+}$ well organize its four strong acidic imidazolium $\mathrm{C}-\mathrm{H}$ bonds for effective small inorganic anion binding mainly via intermolecular hydrogen bonds; meanwhile the bigger cavity and longer distances between the strong acidic imidazolium $\mathrm{C}-\mathrm{H}$ bonds of $\mathbf{1}^{4+}$ leads two possible results: (1) worse cooperation of its acidic $\mathrm{C}-\mathrm{H}$ bonds weaken the intermolecular hydrogen bonding interactions for small anion complexation; (2) its more relax backbone has possible benefits of binding more anion guests. In summary, small distinctions of the backbones between
\end{abstract}

*E-mail:xulj@chem.ruc.edu.cn; hanyuangong@bnu.edu.cn

Received February 27, 2014; revised March 12, 2014; published online April 10, 2014

Project supported by the National Natural Science Foundation of China (Nos. 21202199, 21372258).

国家自然科学基金(Nos. 21202199, 21372258)资助项目。 
$\mathbf{1}^{4+}$ and $\mathbf{2}^{4+}$ result in significantly different anion complexations, including stoichiometries, association constants, binding modes, etc. This finding will help to guide following macrocyclic anion receptor design and study.

Keywords macrocycle; backbone; non-covalent weak interactions; anion binding

超分子化学是研究分子间通过非共价键弱相互作 用所形成的具有特定功能的有序分子聚集体的化 学 ${ }^{[1 \sim 7]}$. 在超分子化学的发展过程中, 大环化合物的研 究发挥了重要的先导作用, 是奠定超分子化学的重要基 础之一, 并促进超分子化学的快速发展 ${ }^{[8,9]}$. 由于预组织 的空腔结构使得分子识别能够在熵效应的作用下有效 进行, 因此针对特定客体分子开展相关大环主体化合物 的设计和研究一直被视为获得高选择性化学感应器的

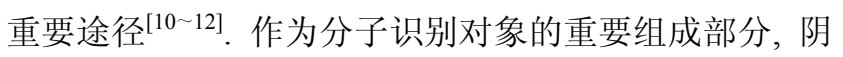
离子在生命科学和环境科学等领域都发挥着不可忽视 的作用, 如磷酸盐是生物体代谢过程的重要产物; 硫酸 氢根离子是酸雨的重要组成等 ${ }^{[11,13]}$. 研究阴离子识别在 化学、生命科学、材料科学、环境科学与信息科学等领 域的应用均具有重要意义 ${ }^{[14]}$. 总体而言, 基于分子间氢 键作用, 针对阴离子客体的大环主体化学感应器可以分 为两类: (1) $\mathrm{N}-\mathrm{H}$ 键为作用位点: 如 Lehn 等 ${ }^{[15 ~ 17]}$ 发展 的氮杂冠醚可以有效结合 $\mathrm{H}_{2} \mathrm{PO}_{4}^{-}$, 进而可作为磷酸酯 水解的模拟酶; Sessler 课题组 ${ }^{[18,19]}$ 发展的杯四吡咯大环 可调整其构象为雉式有效与 $\mathrm{Cl}^{-}$发生相互作用并可应用 于构筑阴离子调控的分子机器; (2) $\mathrm{C}-\mathrm{H}$ 键为作用位点: 这一领域的研究在 2000 年之后才发展起来, 并以带正 电荷的 $\mathrm{C}-\mathrm{H}$ 键的应用为开端. Yoon 及其他的研究 者 ${ }^{[20 ~ 25]}$ 利用咪唑单元合成了一系列阴离子受体, 并详 细研究了这些化合物对部分无机阴离子的识别性能. 从 2002 年开始, Kim 等 ${ }^{[26,27]}$ 报道了一系列含有咪唑基团的 大环主体化合物识别卤素离子的工作, 更通过引入硝 基, 实现了大环主体化合物对 $\mathrm{Cl}^{-}$和 $\mathrm{H}_{2} \mathrm{PO}_{4}^{-}$的识别. 2013 年, You 研究组 ${ }^{[28,29]}$ 合成了含有 4 个咪唑片段的刚 性大环, 发现该大环对硫酸根离子具有显著的识别作 用. 以 $\mathrm{C}-\mathrm{H}$ 键为作用位点的研究还包括中性 $\mathrm{C}-\mathrm{H}$ 键 的应用, 如 Flood 等 ${ }^{[30 ~ 34]}$ 设计合成的大环化合物通过中 性 $\mathrm{C}-\mathrm{H}$ 键作用位点可有效结合无机阴离子客体. $\mathrm{C}-\mathrm{H}$ 键作为阴离子受体的分子间氢键活性作用位点, 受到研 究人员的广泛关注, 主要有两方面的原因: (1) $\mathrm{C}-\mathrm{H}$ 键 广泛存在于自然界, 但直到目前, 对于 $\mathrm{C}-\mathrm{H}$ 键所涉及 的分子间弱相互作用的研究仍较为蒉乏; (2)作为分子间 氢键的活性作用位点, $\mathrm{C}-\mathrm{H}$ 键与 $\mathrm{N}-\mathrm{H}$ 键相比较, 具有 相近的作用能力, 而结构更稳定, 对阴离子发生响应的 pH 范围更广.

咪唑盐结构由于其 2 位 $\mathrm{C}-\mathrm{H}$ 键具有较强的酸性, 能与阴离子形成稳定的分子间氢键, 并且所带的正电荷 同样有利于与阴离子的结合, 因而被广泛地应用于基于
$\mathrm{C}-\mathrm{H}$ 作用的、作为阴离子受体的大环主体化合物的研 究中. 在此前的研究中, 环 [2](2,6-二(1H-咪唑基)吡啶 [2](1,4-二亚甲基苯) $\left(\mathbf{1}^{4+}\right)$ 和环 $[2](2,6-$ 二(1 $H$-咪唑基)吡啶 [2](1,2-二亚甲基苯) $\left(2^{4+}\right)$ 被高效合成 ${ }^{[35,36]}$. 这两者都是 骨架柔顺的四咪唑盐基大环主体化合物. 研究表明, 桥 连基团的改变导致大环分子采取截然不同的构象与阴 离子相互结合: 如对于 $\mathrm{PF}_{6}^{-}, \mathbf{1}^{4+}$ 以船式构象、部分椅式 或全椅式构象与之结合; $\mathbf{2}^{4+}$ 则是采取近于平面或夹式 构象与之作用; 对于 2,6-荎二甲酸的二价阴离子, $\mathbf{1}^{4+}$ 可 调整其构象为 “箱式” 与之形成假轮烷结构, 而 $\mathbf{2}^{4+}$ 通 过近于平面的构象来与之结合, 并进一步在溶液中及固 相中形成层状自组装体系. 但上述大环主体化合物 $\mathbf{1}^{4+}$ 或 $\mathbf{2}^{4+}$ 对于更多阴离子, 尤其是体积较小、与生物科学 和环境科学密切相关的无机阴离子如 $\mathrm{Cl}^{-}, \mathrm{Br}^{-}, \mathrm{I}^{-}, \mathrm{N}_{3}^{-}$, $\mathrm{NO}_{3}^{-}$或 $\mathrm{HSO}_{4}^{-}$的作用仍未加以考察. 本文探讨这两个 大环化合物由于桥连基团的不同, 所引起的骨架结构和 构象调控的差异对于阴离子识别的影响, 以利于今后新 型大环阴离子感应器的设计和研究.

\section{1 结果与讨论}

为了深入研究所合成的大环主体化合物 $\mathbf{1}^{4+}$ 及 $\mathbf{2}^{4+}$ 作为阴离子受体的作用机制, 探讨它们骨架结构的差异 在与阴离子发生相互作用时的影响, 使之在后期的研究 中发挥先导作用, 详细研究了主客体之间的相互作用 (图 1).

两个大环主体化合物的骨架结构仅在连接基团有 所差别: $\mathbf{1}^{4+}$ 的桥连基团是 1,4-二亚甲基苯基; 而 $\mathbf{2}^{4+}$ 的桥 连基团是 1,2-二亚甲基苯基 ${ }^{[36]}$. 在前期的工作中已经发 现这两个大环主体化合物由于结构的不同而使得它们 与 2,6 - 萗二甲酸的二价阴离子 $(3)$ 之间有着截然不同的 结合方式: $\mathbf{1}^{4+}$ 能够有效地与之形成假轮烷的结构, 并能 作为前体在加入金属离子时进一步形成三维的轮烷超 分子有机骨架结构体系(RSOFs)或是一维至三维的具有 轮烷结构的金属有机骨架体系 ${ }^{[37,38]} ; \mathbf{2}^{4+}$ 则只能够通过扭 曲的椅式构象与 3 形成层状的自组装结构 ${ }^{[36]}$. 但对上述 大环主体化合物与丰富种类阴离子间的相互作用仍需 细致研究, 以深入认识大环主体化合物骨架结构对其与 客体阴离子之间相互作用的影响. 因此在本工作中, 对 上述主体 $\mathbf{1}^{4+}$ 或 $\mathbf{2}^{4+}$ 与图示一系列阴离子客体的相互作 用进行了详细探讨，这些客体阴离子包括球型的卤素离 子 $\mathrm{Cl}^{-}, \mathrm{Br}^{-}, \mathrm{I}^{-}$; 线型的 $\mathrm{N}_{3}^{-}$; 三角型的 $\mathrm{NO}_{3}^{-}$以及四面体 型的 $\mathrm{HSO}_{4}^{-}$, 以充分探讨大环骨架结构对大环主体分子 

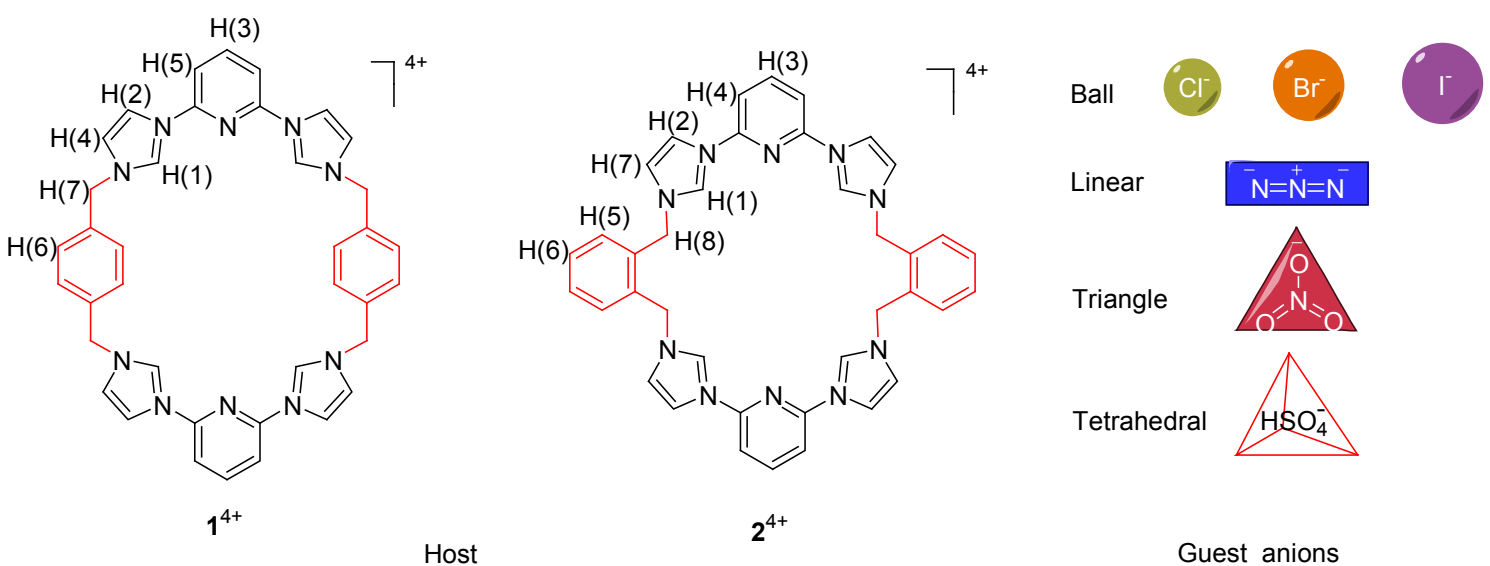

图 1 大环主体化合物 $\mathbf{1}^{4+}$ 及 $\mathbf{2}^{4+}$ 和不同形状的阴离子客体的结构示意图

Figure 1 Structures of macrocyclic hosts $\mathbf{1}^{4+}$ or $2^{4+}$ and anionic guests with different shapes

和客体阴离子相互作用的影响, 希望能够通过以上研究 深入探讨大环阴离子感应器设计的一般规律.

\section{1 溶液中主客体相互作用的化学计量比测定}

主客体之间形成稳定复合物时的化学计量比通过 核磁 ${ }^{1} \mathrm{H}$ 谱的 Job's plot 得以确定 ${ }^{[39,40]}$. 溶液中的主客体 相互作用研究均在 $d_{6}$-DMSO 中进行 (如图 2, 图 3 所示).

$\mathbf{1}^{4+}$ 与所研究的阴离子客体间进行核磁 ${ }^{1} \mathrm{H}$ 谱 Job's plot 的测定时, 主客体浓度之和维持如下: $[H]+[G]=2$ $\mathrm{mmol} \cdot \mathrm{L}^{-1}\left(\mathrm{H}\right.$ : 大环主体化合物 $\mathbf{1}^{4+}$; $\mathrm{G}$ : 客体阴离子为 $\mathrm{Br}^{-}, \mathrm{I}^{-}, \mathrm{N}_{3}^{-}, \mathrm{NO}_{3}^{-}, \mathrm{HSO}_{4}^{-}$)或 $[\mathrm{H}]+[\mathrm{G}]=1 \mathrm{mmol} \cdot \mathrm{L}^{-1}$ $\left(\mathrm{G}\right.$ : 客体阴离子为 $\left.\mathrm{Cl}^{-}\right)$; 而对于 $\mathbf{2}^{4+}$, 则在实验时保持 $[\mathrm{H}]+[\mathrm{G}]=2 \mathrm{mmol} \cdot \mathrm{L}^{-1}\left(\mathrm{H}\right.$ : 大环主体化合物 $\mathbf{2}^{4+} ; \mathrm{G}$ : 客 体阴离子为 $\mathrm{Cl}^{-}, \mathrm{Br}^{-}, \mathrm{I}^{-}, \mathrm{N}_{3}^{-}, \mathrm{HSO}_{4}^{-}$)或者 $[\mathrm{H}]+[\mathrm{G}]=$ $10 \mathrm{mmol} \cdot \mathrm{L}^{-1}\left(\mathrm{G}: \mathrm{NO}_{3}^{-}\right)$. 如图 2, 图 3 所示, $\mathbf{1}^{4+}$ 与客体 阴离子 $\mathrm{Cl}^{-}, \mathrm{Br}^{-}, \mathrm{I}^{-}$或 $\mathrm{NO}_{3}^{-}$相互作用的 Job's plot 最大值 为 0.67 , 表明以上各对主客体间化学计量比均为 $1: 2$ $([\mathrm{H}]$ : $[\mathrm{G}])$; 而当阴离子客体为 $\mathrm{HSO}_{4}^{-}$或 $\mathrm{N}_{3}^{-}, \mathbf{1}^{4+}$ 与之发 生作用的 Job's plot 显示最大值为 0.6 , 意味着此时主客 体化学计量比为 $2: 3([\mathrm{H}]:[\mathrm{G}]) ;$ 与 $\mathbf{1}^{4+}$ 不同, $\mathbf{2}^{4+}$ 与 $\mathrm{Cl}^{-}$, $\mathrm{Br}^{-}, \mathrm{N}_{3}^{-}$或 $\mathrm{HSO}_{4}^{-}$相互作用的 Job's plot 峰值为 0.5 , 表 明它们主客体相互作用的化学计量比均为 $1: 1([\mathrm{H}]$ : $[G]) ; 2^{4+}$ 与 $\mathrm{NO}_{3}{ }^{-}$或 $\mathrm{I}^{-}$的 Job's plot 曲线的最大值均位于 0.6 , 意味着相应化学计量比是 $2: 3([\mathrm{H}]:[\mathrm{G}])$. 综合上 述结果, 发现在所考察的体系中, 对于同一客体阴离子, 存在化学计量比 $([\mathrm{H}]:[\mathrm{G}])$ 的值总有 $\mathbf{1}^{4+} \geqslant \mathbf{2}^{4+}$ 的关系, 即等量的 $\mathbf{1}^{4+}$ 比 $\mathbf{2}^{4+}$ 能结合更多的客体阴离子. 推测发生 上述现象的可能原因是桥连基团的不同使得 $\mathbf{1}^{4+}$ 与阴离 子能够有效结合的 $\mathrm{C}-\mathrm{H}$ 键作用位点相对分散, 更易于 与更多体积较小的客体阴离子以较弱的分子间氢键作 用相结合; 而 $\mathbf{2}^{4+}$ 的 $\mathrm{C}-\mathrm{H}$ 键作用位点相对集中, 更易于 协同与相对少量的阴离子客体进行更稳定的结合.

\section{2 溶液中主客体形成复合物时结合常数的测定}

为了进一步考察上述主客体之间的相互作用，核磁 ${ }^{1} \mathrm{H}$ 谱滴定实验被详细研究以获得主客体相互作用的结 合常数. 在滴定过程中维持大环主体化合物的浓度 $(0.5$ $\mathrm{mmol} \cdot \mathrm{L}^{-1}$ )不变, 发现其核磁 ${ }^{1} \mathrm{H}$ 谱信号随客体浓度的逐 步增加而产生化学位移的渐变. 对同一客体 $\mathrm{N}_{3}^{-}$离子, $1^{4+}$ 和 $2^{4+}$ 与之进行主客体相互作用时所获得的滴定图谱 作为本文研究中所获得图谱的示例如图 4 .

如图 4 及图 5a 所示, 大环化合物 $\mathbf{1}^{4+}$ 在与 $\mathrm{N}_{3}^{-}$发生 相互作用时, 酸性最强的咪唑基 2 位 $\mathrm{C}-\mathrm{H}$ 键 $[\mathrm{H}(1)]$ 受到 客体诱导所产生的低场化学位移在 $\mathbf{1}^{4+}$ 中比在 $\mathbf{2}^{4+}$ 中要 小很多(当主体浓度均为 $0.5 \mathrm{mmol} \cdot \mathrm{L}^{-1}, \mathrm{~N}_{3}^{-}$浓度均为为 $2 \mathrm{mmol} \cdot \mathrm{L}^{-1}$ 时，它们之间的差值达到 $300 \mathrm{~Hz}$ ), 表明此 时 $\mathbf{1}^{4+}$ 比 $2^{4+}$ 与阴离子形成分子间氢键的能力要弱得多. 而如图 4 及图 $5 \mathrm{~b}$ 所示, 大环化合物 $\mathbf{1}^{4+}$ 在与 $\mathrm{N}_{3}^{-}$发生相 互作用时, 位于吡啶环上的 4 位 $\mathrm{C}-\mathrm{H}[\mathrm{H}(3)]$, 仅有 $\mathbf{1}^{4+}$ 产生了向高场的位移, 而 $\mathbf{2}^{4+}$ 仍然呈现作为氢键受体的 向低场的位移，表明在与 $\mathrm{N}_{3}^{-}$发生相互作用的过程当中， $1^{4+}$ 能够较为有效地与之发生 anion- $\pi$ 的相互作用, 而 $2^{4+}$ 则主要表现分子间氢键作用.

上述结果充分体现了大环主体化合物的骨架结构 对于阴离子结合模式的重要影响. 除了如图所示的 $\mathrm{N}_{3}^{-}$ 之外, 所考察的其他离子 $\left(\mathrm{Cl}^{-}, \mathrm{Br}^{-}, \mathrm{I}^{-}, \mathrm{N}_{3}^{-}, \mathrm{NO}_{3}^{-}\right.$或 $\mathrm{HSO}_{4}^{-}$) 也对主体分子 $\mathbf{1}^{4+}$ 或 $\mathbf{2}^{4+}$ 的质子信号有类似影响, 表明骨架结构对于主客体相互作用的影响具有广泛的 意义: 将作用位点进行针对目标客体的预组织, 将使主 客体之间的作用达到最大匹配，达到最优化的主客体分 子识别效果.

综合前述溶液中的研究，根据 ${ }^{1}$ H NMR Job's plots 的结果推断主客体复合的平衡模式, 应用 Hyperquad 2003 软件, 考察大环主体化合物 $\mathbf{1}^{4+}$ 上的质子信号的化 


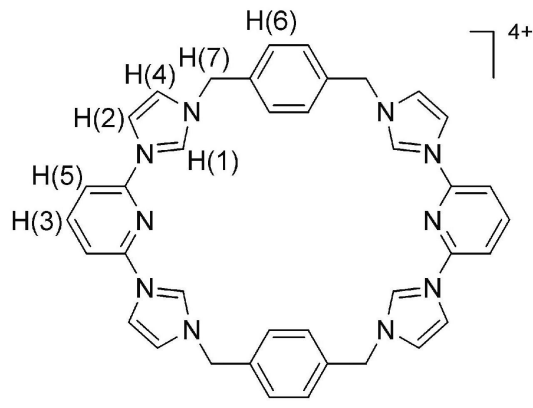

$1^{4+}$
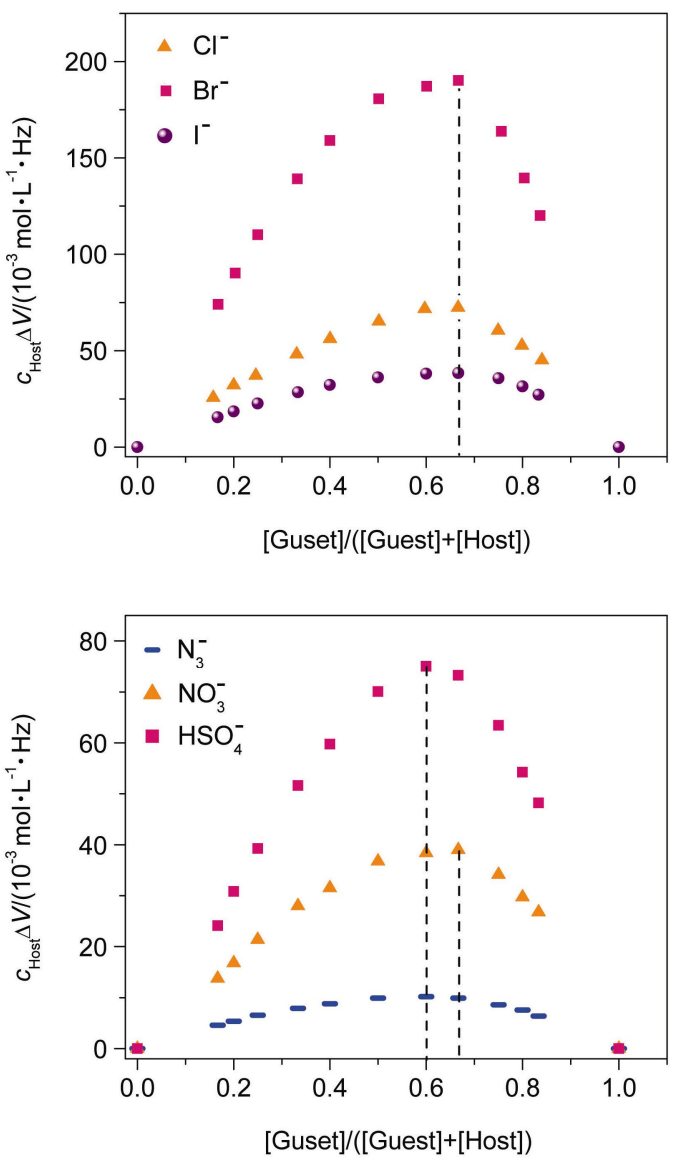

图 2 大环主体化合物 $\mathbf{1}^{4+}$ 和阴离子客体相互作用的核磁 ${ }^{1} \mathrm{H}$ 谱 Job's plots 图

Figure $2{ }^{1} \mathrm{H}$ NMR Job's plots corresponding to the bindings between host $\mathbf{1}^{4+} \cdot 4 \mathrm{PF}_{6}{ }^{-}$and anionic guests

学位移改变值与对应的客体浓度之间的函数关系, 曲线 拟合得到大环主体化合物 $\mathbf{1}^{4+}$ 和 $\mathbf{2}^{4+}$ 与所考察的无机阴 离子物种间的 $K_{\mathrm{a} 1}$ 或 $K_{\mathrm{a} 2}$ 值 ${ }^{[39 \sim 41]}$, 将结果归纳如表 1 所 示.

综合所有 Job's plot 研究、滴定及相关计算的结果, 可以发现: (1)在同样的条件下, 主客体之间形成稳定复 合物时, 与 $2^{4+}$ 相比较, $\mathbf{1}^{4+}$ 总是能够结合等量或者更多 的同种阴离子客体. (2)结合常数的比较: 尽管主客体相 互作用的不同平衡其结合常数难以比较, 但主客体形成

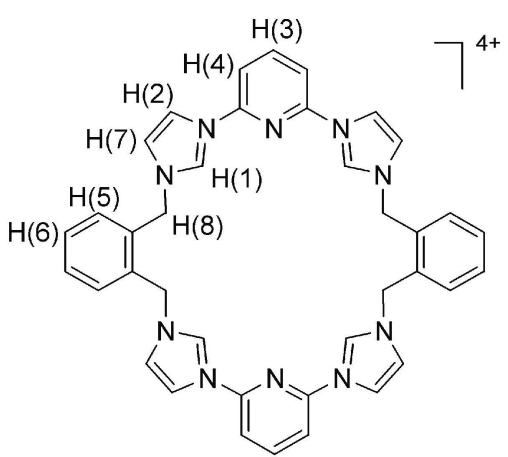

$2^{4+}$
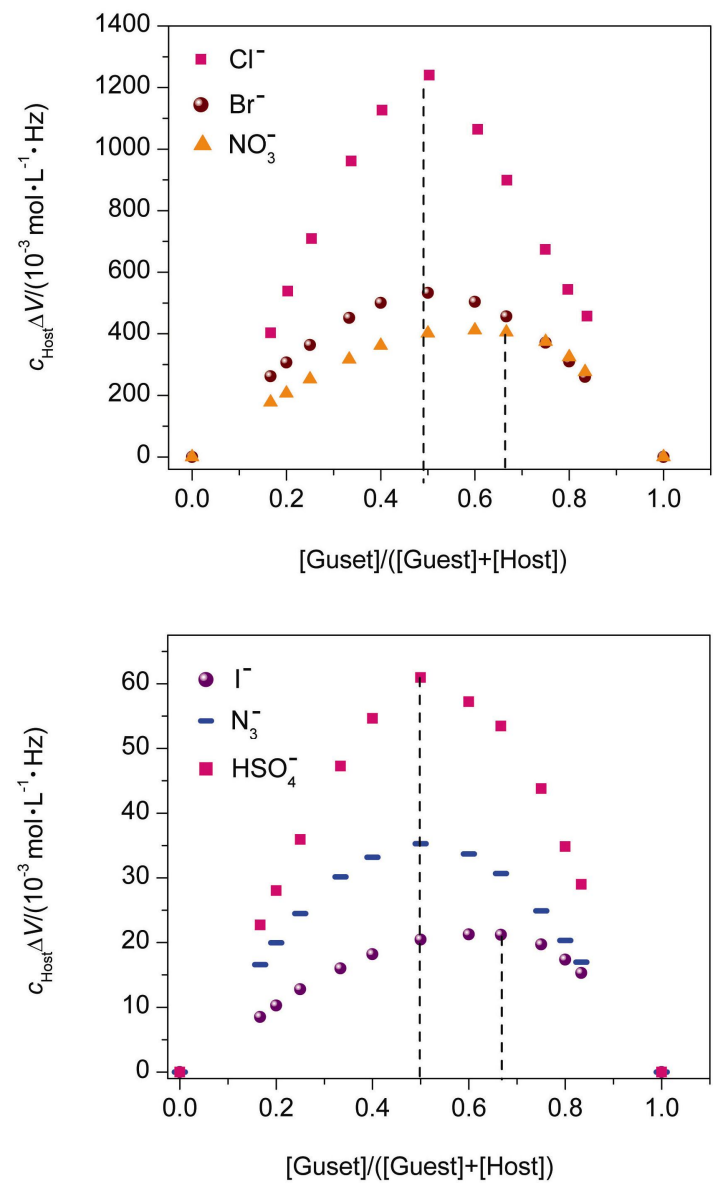

图 3 大环主体化合物 $\mathbf{2}^{4+}$ 和阴离子客体相互作用的核磁 ${ }^{1} \mathrm{H}$ 谱 Job's plots 图

Figuer $3{ }^{1} \mathrm{H}$ NMR Job'splots corresponding to the bindings between host $\mathbf{2}^{4+} \cdot 4 \mathrm{PF}_{6}^{-}$and anionic guests

$1: 1$ 复合物的结合常数仍可作为二者与阴离子相互作 用强弱的指标：对于易于形成分子间氢键与大环主体进 行复合的阴离子即 $\mathrm{Cl}^{-}, \mathrm{N}_{3}^{-}, \mathrm{NO}_{3}^{-}, \mathrm{HSO}_{4}^{-}, 2^{4+}$ 与该类 阴离子的结合常数 $\left(K_{\mathrm{a} 1}\right)$ 总是大于甚至是远大于 $\mathbf{1}^{4+}$. 但 是对于较难形成分子间氢键，随着离子半径的增大导致 极化性增强, 更易于发生 anion- $\pi$ 作用的离子如 $\mathrm{Br}^{-}$和 $\mathrm{I}^{-}, \mathbf{1}^{4+}$ 与它们的结合常数近于甚至大于 $2^{4+}$. 
表 1 主客体复合的热力学平衡状态、结合常数的总结

Table 1 Summary of equilibrium and the calculated association constants $K_{\mathrm{a}}$

\begin{tabular}{|c|c|c|c|c|c|}
\hline \multicolumn{2}{|c|}{ Guest } & \multirow{2}{*}{ Host } & \multirow{2}{*}{ Host/Guest } & \multirow{2}{*}{ Equilibrium } & \multirow{2}{*}{ Association constants $K_{\mathrm{a}}$} \\
\hline Shape & Species & & & & \\
\hline \multirow{6}{*}{ Ball } & \multirow[t]{2}{*}{$\mathrm{Cl}^{-}$} & $\mathbf{1}^{4+}$ & $1: 2$ & $\begin{array}{l}{[\mathrm{H}]+[\mathrm{G}] \stackrel{K_{\mathrm{a} 1}}{\rightleftharpoons}[\mathrm{HG}]} \\
{[\mathrm{HG}]+[\mathrm{G}] \stackrel{K_{\mathrm{a} 2}}{\rightleftharpoons}\left[\mathrm{HG}_{2}\right]}\end{array}$ & $\begin{array}{l}K_{\mathrm{a} 1}=(4.2 \pm 0.2) \times 10^{3}{\mathrm{~L} \cdot \mathrm{mol}^{-1}}^{-1} \\
K_{\mathrm{a} 2}=(6.2 \pm 0.6) \times 10^{2}{\mathrm{~L} \cdot \mathrm{mol}^{-1}}^{-1}\end{array}$ \\
\hline & & $2^{4+}$ & $1: 1$ & {$[\mathrm{H}]+[\mathrm{G}] \stackrel{K_{\mathrm{a}}}{\rightleftharpoons}[\mathrm{HG}]$} & $K_{\mathrm{a}}=(1.0 \pm 0.1) \times 10^{5} \mathrm{~L} \cdot \mathrm{mol}^{-1}$ \\
\hline & \multirow[t]{2}{*}{$\mathrm{Br}^{-}$} & $1^{4+}$ & $1: 2$ & $\begin{array}{l}{[\mathrm{H}]+[\mathrm{G}] \stackrel{K_{\mathrm{a} 1}}{\rightleftharpoons}[\mathrm{HG}]} \\
{[\mathrm{HG}]+[\mathrm{G}] \stackrel{K_{\mathrm{a} 2}}{\rightleftharpoons}\left[\mathrm{HG}_{2}\right]}\end{array}$ & $\begin{array}{l}K_{\mathrm{a} 1}=(2.7 \pm 0.1) \times 10^{4} \mathrm{~L} \cdot \mathrm{mol}^{-1} \\
K_{\mathrm{a} 2}=(4.3 \pm 0.4) \times 10^{2}{\mathrm{~L} \cdot \mathrm{mol}^{-1}}^{-1}\end{array}$ \\
\hline & & $2^{4+}$ & $1: 1$ & {$[\mathrm{H}]+[\mathrm{G}] \stackrel{K_{\mathrm{a}}}{\rightleftharpoons}[\mathrm{HG}]$} & $K_{\mathrm{a}}=(2.0 \pm 0.1) \times 10^{4} \mathrm{~L} \cdot \mathrm{mol}^{-1}$ \\
\hline & \multirow{2}{*}{$\mathrm{I}^{-}$} & $\mathbf{1}^{4+}$ & $1: 2$ & $\begin{array}{l}{[\mathrm{H}]+[\mathrm{G}] \stackrel{K_{\mathrm{a} 1}}{\rightleftharpoons}[\mathrm{HG}]} \\
{[\mathrm{HG}]+[\mathrm{G}] \stackrel{K_{\mathrm{a} 2}}{\rightleftharpoons}\left[\mathrm{HG}_{2}\right]}\end{array}$ & $\begin{array}{l}K_{\mathrm{a} 1}=(5.9 \pm 0.2) \times 10^{3}{\mathrm{~L} \cdot \mathrm{mol}^{-1}}^{-1} \\
K_{\mathrm{a} 2}=(2.0 \pm 0.3) \times 10^{2}{\mathrm{~L} \cdot \mathrm{mol}^{-1}}^{-1}\end{array}$ \\
\hline & & $2^{4+}$ & $2: 3$ & $\begin{array}{l}{[\mathrm{H}]+[\mathrm{G}] \stackrel{K_{\mathrm{a} 1}}{\rightleftharpoons}[\mathrm{HG}]} \\
2[\mathrm{HG}]+[\mathrm{G}] \stackrel{K_{\mathrm{a} 2}}{\rightleftharpoons}\left[\mathrm{H}_{2} \mathrm{G}_{3}\right]\end{array}$ & $\begin{array}{l}K_{\mathrm{a} 1}=(8.9 \pm 0.1) \times 10^{2} \mathrm{~L} \cdot \mathrm{mol}^{-1} \\
K_{\mathrm{a} 2}=(5.1 \pm 0.5) \times 10^{8}\left(\mathrm{~mol} \cdot \mathrm{L}^{-1}\right)^{-2}\end{array}$ \\
\hline \multirow[t]{2}{*}{ Linear } & \multirow[t]{2}{*}{$\mathrm{N}_{3}^{-}$} & $\mathbf{1}^{4+}$ & $2: 3$ & $\begin{array}{l}{[\mathrm{H}]+[\mathrm{G}] \stackrel{K_{\mathrm{a} 1}}{\rightleftharpoons}[\mathrm{HG}]} \\
2[\mathrm{HG}]+[\mathrm{G}] \stackrel{K_{\mathrm{a} 2}}{\rightleftharpoons}\left[\mathrm{H}_{2} \mathrm{G}_{3}\right]\end{array}$ & $\begin{array}{l}K_{\mathrm{a} 1}=(2.8 \pm 0.1) \times 10^{2}{\mathrm{~L} \cdot \mathrm{mol}^{-1}}^{-1} \\
K_{\mathrm{a} 2}=(1.6 \pm 0.2) \times 10^{7}\left(\mathrm{~mol} \cdot \mathrm{L}^{-1}\right)^{-2}\end{array}$ \\
\hline & & $2^{4+}$ & $1: 1$ & {$[\mathrm{H}]+[\mathrm{G}] \stackrel{K_{\mathrm{a}}}{\rightleftharpoons}[\mathrm{HG}]$} & $K_{\mathrm{a}}=(4.8 \pm 0.1) \times 10^{3}{\mathrm{~L} \cdot \mathrm{mol}^{-1}}^{-1}$ \\
\hline \multirow{2}{*}{ Triangle } & \multirow[b]{2}{*}{$\mathrm{NO}_{3}^{-}$} & $\mathbf{1}^{4+}$ & $1: 2$ & $\begin{array}{l}{[\mathrm{H}]+[\mathrm{G}] \stackrel{K_{\mathrm{a} 1}}{\rightleftharpoons}[\mathrm{HG}]} \\
{[\mathrm{HG}]+[\mathrm{G}] \stackrel{K_{\mathrm{a} 2}}{\rightleftharpoons}\left[\mathrm{HG}_{2}\right]}\end{array}$ & $\begin{array}{l}K_{\mathrm{a} 1}=(2.0 \pm 0.1) \times 10^{2}{\mathrm{~L} \cdot \mathrm{mol}^{-1}}^{-1} \\
K_{\mathrm{a} 2}=(6.3 \pm 0.3) \times 10^{0}{\mathrm{~L} \cdot \mathrm{mol}^{-1}}^{-1}\end{array}$ \\
\hline & & $2^{4+}$ & $2: 3$ & $\begin{array}{l}{[\mathrm{H}]+[\mathrm{G}] \stackrel{K_{\mathrm{a} 1}}{\rightleftharpoons}[\mathrm{HG}]} \\
2[\mathrm{HG}]+[\mathrm{G}] \stackrel{K_{\mathrm{a} 2}}{\rightleftharpoons}\left[\mathrm{H}_{2} \mathrm{G}_{3}\right]\end{array}$ & $\begin{array}{l}K_{\mathrm{a} 1}=(6.8 \pm 0.2) \times 10^{2}{\mathrm{~L} \cdot \mathrm{mol}^{-1}}^{-1} \\
K_{\mathrm{a} 2}=(9.4 \pm 0.3) \times 10^{3}\left(\mathrm{~mol} \cdot \mathrm{L}^{-1}\right)^{-2}\end{array}$ \\
\hline \multirow[t]{2}{*}{ Tetrahedral } & \multirow[t]{2}{*}{$\mathrm{HSO}_{4}^{-}$} & $\mathbf{1}^{4+}$ & $2: 3$ & $\begin{array}{l}{[\mathrm{H}]+[\mathrm{G}] \stackrel{K_{\mathrm{a} 1}}{\rightleftharpoons}[\mathrm{HG}]} \\
2[\mathrm{HG}]+[\mathrm{G}] \stackrel{K_{\mathrm{a} 2}}{\rightleftharpoons}\left[\mathrm{H}_{2} \mathrm{G}_{3}\right]\end{array}$ & $\begin{array}{l}K_{\mathrm{a} 1}=(8.3 \pm 0.3) \times 10^{2}{\mathrm{~L} \cdot \mathrm{mol}^{-1}}^{-1} \\
K_{\mathrm{a} 2}=(6.1 \pm 0.4) \times 10^{7}\left(\mathrm{~mol} \cdot \mathrm{L}^{-1}\right)^{-2}\end{array}$ \\
\hline & & $2^{4+}$ & $1: 1$ & {$[\mathrm{H}]+[\mathrm{G}] \stackrel{K_{\mathrm{a}}}{\rightleftharpoons}[\mathrm{HG}]$} & $K_{\mathrm{a}}=(9.2 \pm 0.1) \times 10^{3}{\mathrm{~L} \cdot \mathrm{mol}^{-1}}^{-1}$ \\
\hline
\end{tabular}

推测产生以上结果的原因是 $\mathbf{2}^{4+}$ 由于其紧凑的骨架 结构使 4 个酸性较强的咪唑盐基 2 位 $\mathrm{C}-\mathrm{H}$ 位点能够有 效协同与体积较小的阴离子同时形成强的分子间氢键; 而 $\mathbf{1}^{4+}$ 的骨架结构使得上述位点的空间距离较大，难以 协同，最终结果是等量 $\mathbf{2}^{4+}$ 只能结合较少的该类阴离子，
主客体为 $1: 1$ 时结合常数总是大于甚至是远大于 $\mathbf{1}^{4+}$. 但是对于较难形成分子间氢键，但更易于发生 anion- $\pi$ 作用的离子如 $\mathrm{Br}^{-}$和 $\mathrm{I}^{-}$，由于 $\mathbf{1}^{4+}$ 更易于采取立体空腔， 在以氢键结合阴离子的同时进行 anion- $\pi$ 作用，使得 $\mathbf{1}^{4+}$ 与它们的 $1: 1$ 结合常数近于甚至大于 $\mathbf{2}^{4+}$. 以上推测获 


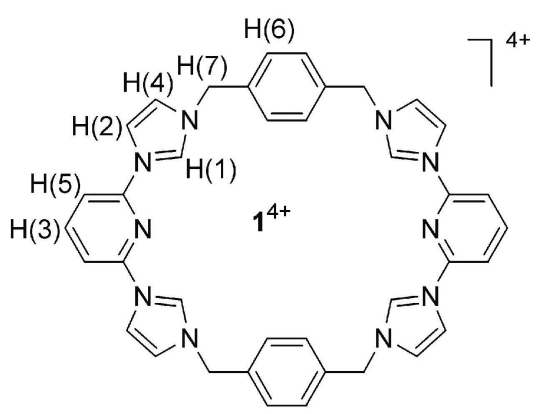

guest/host

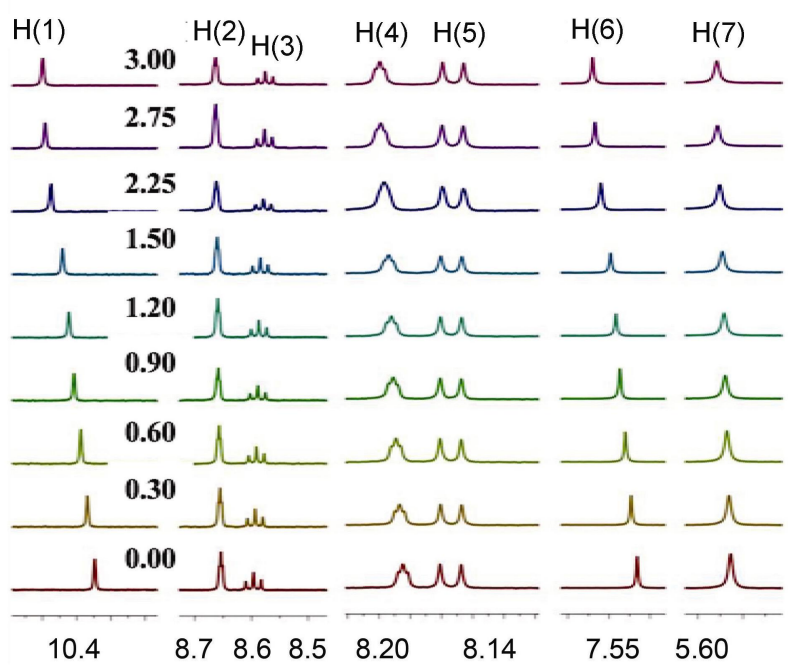

b

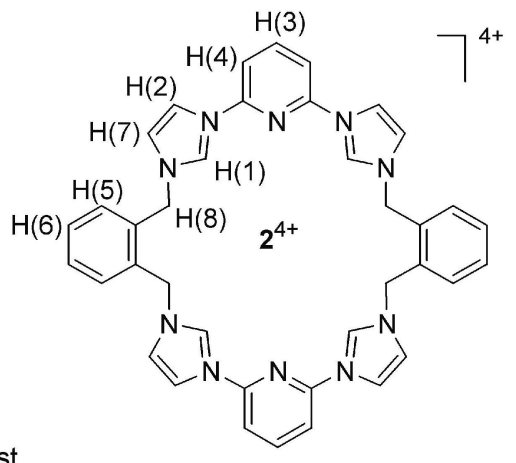

guest/host

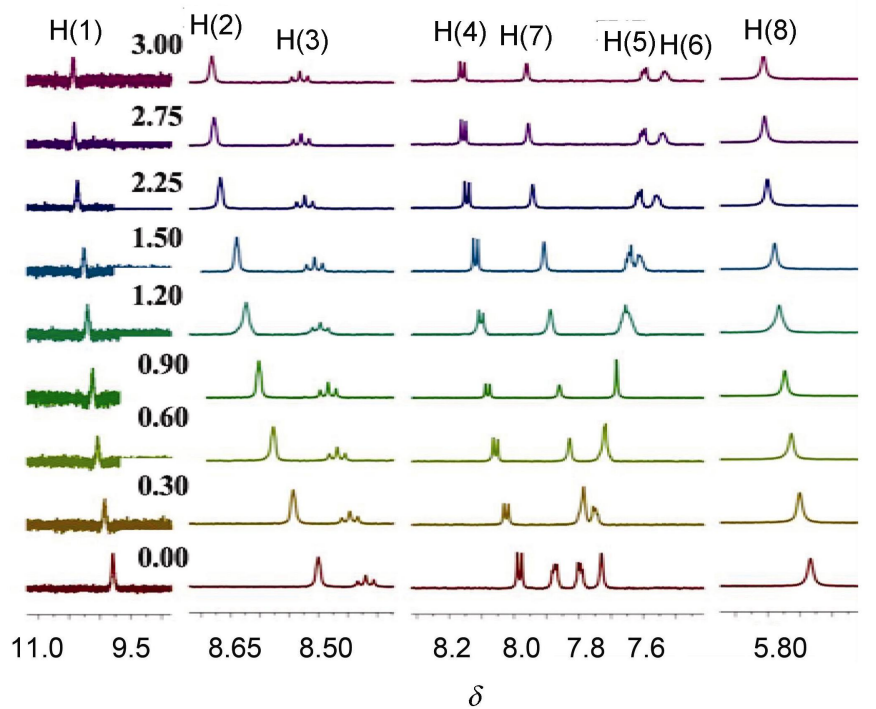

图 4 大环主体化合物 $\mathbf{1}^{4+}\left(5.00 \times 10^{-4} \mathrm{~mol} \cdot \mathrm{L}^{-1}\right)$ 和 $\mathrm{N}_{3}^{-}$的核磁 ${ }^{1} \mathrm{H}$ 谱滴定图(a)与 $\mathbf{2}^{4+}\left(5.00 \times 10^{-4} \mathrm{~mol} \cdot \mathrm{L}^{-1}\right)$ 和 $\mathrm{N}_{3}^{-}$的核磁 ${ }^{1} \mathrm{H}$ 谱滴定 图(b)

Figure $4{ }^{1} \mathrm{H}$ NMR spectroscopic titrations of $\mathbf{1}^{4+}\left(5.00 \times 10^{-4} \mathrm{~mol} \cdot \mathrm{L}^{-1}\right)(\mathrm{a})$ or $\mathbf{2}^{4+}\left(5.00 \times 10^{-4} \mathrm{~mol} \cdot \mathrm{L}^{-1}\right)$ (b) with increasing $\mathrm{N}_{3}^{-}$
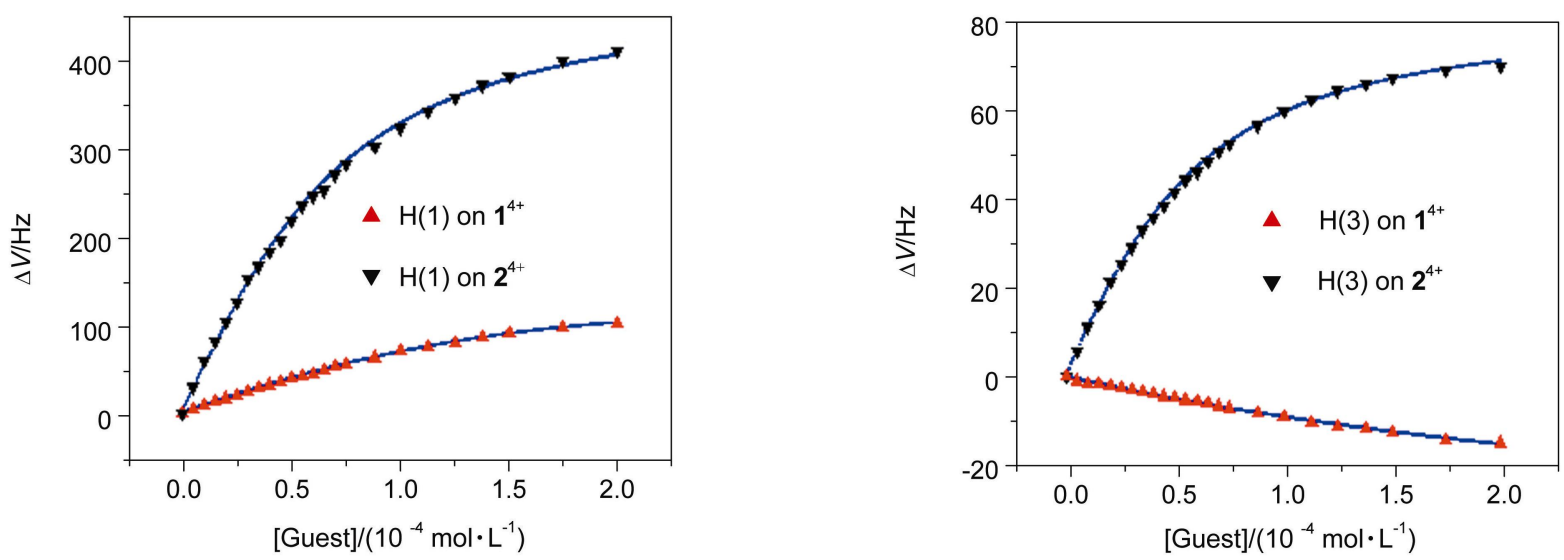

图 $5 \mathbf{1}^{4+}$ 或 $\mathbf{2}^{4+}$ 的 $\mathrm{H}(1)$ 信号(a)及 $\mathrm{H}(3)$ 的信号(b)随着阴离子客体 $\mathrm{N}_{3}^{-}$浓度的增加而发生改变

Figure $5{ }^{1} \mathrm{H}$ NMR binding isotherms about $\mathrm{H}(1)$ (a), H(3) (b) on $\mathbf{1}^{4+}$ or $\mathbf{2}^{4+}$ associated with additional $\mathrm{N}_{3}^{-}$ The dash lines show the non-linear curve fit of the experiment data to the appropriate equation

得了单晶结构研究的验证.

\section{$1.31^{4+}$ 和 $2^{4+}$ 与阴离子的质谱研究}

采用电喷雾电离质谱进一步研究了气相中主客体 相互作用. 如表 2 所示, 结果表明气相中大环主体化合
物与阴离子客体也存在相互作用.

\section{$1.41^{4+}$ 和 $2^{4+}$ 和部分阴离子的单晶结构图}

单晶结构 $\left[\mathbf{1}^{4+} \cdot 2 \mathrm{PF}_{6}^{-} \cdot 2 \mathrm{NO}_{3}^{-} \cdot 10 \mathrm{H}_{2} \mathrm{O}\right](\mathrm{CCDC}$ No. 979250)和 $\left[\mathbf{1}^{4+} \cdot 4 \mathrm{HSO}_{4}^{-} \cdot \mathrm{H}_{2} \mathrm{O}\right](\mathrm{CCDC}$ No. 979249)通过 
实验部分所描述的方法培养并进行测定, 相关参数如表 3 所示. $\mathrm{CCDC}$ 编号为 842434 的晶体 $\left[\mathbf{2}^{4+} \cdot 2 \mathrm{PF}_{6}^{-}\right.$. $2 \mathrm{H}_{2} \mathrm{PO}_{4}^{-} \cdot 9.5 \mathrm{H}_{2} \mathrm{O}$ ], 参数见文献[36].

如图 6 所示, 在 $\left[\mathbf{1}^{4+} \cdot 2 \mathrm{PF}_{6}^{-} \cdot 2 \mathrm{NO}_{3}^{-} \cdot 10 \mathrm{H}_{2} \mathrm{O}\right]$ 晶体结 构中, $\mathrm{PF}_{6}^{-}$[含原子 $\mathrm{P}(1)$ 与 $\mathbf{1}^{4+}$ 主要通过 $\mathrm{C}(4)$ 与 $\mathrm{C}(12)$ 上 的质子与之发生分子间氢键相互作用, 而 $\mathrm{C}(4 \mathrm{a})$ 与 $\mathrm{C}(12 \mathrm{a})$ 上的氢原子由于距离太远无法与该阴离子发生相 互作用，而与该阴离子具有中心对称性的另外一个 $\mathrm{PF}_{6}^{-}$阴离子发生相互作用.
图 7 所示的 $\left[2^{4+} \cdot 4 \mathrm{PF}_{6}^{-} \cdot 2 \mathrm{H}_{2} \mathrm{PO}_{4}^{-} \cdot 9.5 \mathrm{H}_{2} \mathrm{O}\right]$ 晶体结构 中, $2^{4+}$ 调整其构象为夹式, 4 个酸性较强的咪唑盐 $\mathrm{C}-\mathrm{H}$ 作用位点协同通过强分子间氢键结合 1 个 $\mathrm{PF}_{6}^{-}$. 即在和 同一种类阴离子客体 $\left(\mathrm{PF}_{6}^{-}\right)$结合时, $\mathbf{1}^{4+}$ 可以结合更多的 客体，但 $\mathrm{C}-\mathrm{H}$ 键无法协同，作用较弱; $2^{4+}$ 则调整其构 象，可使所有的 $\mathrm{C}-\mathrm{H}$ 键都参与到同一客体阴离子的复 合作用中，具有更强氢键作用的同时只能结合较少的阴 离子客体. 推测在与其它体积较小的无机阴离子发生相 互作用时，这样的骨架效应同样存在.

表 2 气相质谱结果总结

Table 2 Summary of ESI-MS results.

\begin{tabular}{|c|c|c|c|c|c|}
\hline Compd. & Observed $\mathrm{m} / \mathrm{z}$ value & Peak assignment & Compd. & Observed $\mathrm{m} / \mathrm{z}$ value & Peak assignment \\
\hline $\mathbf{1}^{4+} \cdot 4 \mathrm{Cl}^{-}$ & $\begin{array}{l}222.1 \\
363.3\end{array}$ & $\begin{array}{l}{\left[\mathbf{1}^{4+}+\mathrm{Cl}^{-}+\mathrm{H}\right]^{3+\bullet}} \\
{\left[\mathbf{1}^{4+}+2 \mathrm{Cl}^{-}+\mathrm{Na}+3 \mathrm{H}\right]^{2+\cdot}}\end{array}$ & $2^{4+} \cdot 4 \mathrm{Cl}^{-}$ & $\begin{array}{l}222.1 \\
699.3\end{array}$ & $\begin{array}{l}{\left[2^{4+}+\mathrm{Cl}^{-}+\mathrm{H}\right]^{3+\cdot}} \\
{\left[2^{4+}+2 \mathrm{Cl}^{-}-\mathrm{H}\right]^{+\cdot}}\end{array}$ \\
\hline $\mathbf{1}^{4+} \cdot 4 \mathrm{Br}^{-}$ & 395.4 & {$\left[1^{4+}+2 \mathrm{Br}^{-}+3 \mathrm{H}\right]^{2+\bullet}$} & $2^{4+} \cdot 4 \mathrm{Br}^{-}$ & 402.4 & {$\left[2^{4+}+2 \mathrm{Br}^{-}+\mathrm{Na}-5 \mathrm{H}\right]^{2+\cdot}$} \\
\hline $1^{4+} \cdot 4 \mathrm{I}^{-}$ & $\begin{array}{l}378.3 \\
442.4\end{array}$ & $\begin{array}{l}{\left[\mathbf{1}^{4+}+\mathrm{I}^{-}\right]^{2+}} \\
{\left[\mathbf{1}^{4+}+2 \mathrm{I}^{-}+\mathrm{H}\right]^{2+}}\end{array}$ & $2^{4+} \cdot 4 \mathrm{I}^{-}$ & $\begin{array}{l}252.5 \\
817.5\end{array}$ & $\begin{array}{l}{\left[2^{4+}+\mathrm{I}^{-}\right]^{3+}} \\
{\left[\left(2^{4+}\right)_{2}+3 \mathrm{I}^{-}-6 \mathrm{H}\right]^{2+\cdot}}\end{array}$ \\
\hline $\mathbf{1}^{4+} \cdot 4 \mathrm{~N}_{3}^{-}$ & $\begin{array}{l}347.9 \\
690.4 \\
460.4\end{array}$ & $\begin{array}{l}{\left[\mathbf{1}^{4+}+\mathrm{N}_{3}^{-}+\mathrm{Na}\right]^{2+}} \\
{\left[\left(\mathbf{1}^{4+}\right)_{2}+3 \mathrm{~N}_{3}^{-}-6 \mathrm{H}\right]^{2+}} \\
{\left[\left(\mathbf{1}^{4+}\right)_{2}+3 \mathrm{~N}_{3}^{-}-5 \mathrm{H}\right]^{3+\bullet}}\end{array}$ & $\mathbf{2}^{4+} \cdot 4 \mathrm{~N}_{3}^{-}$ & 716.2 & {$\left[2^{4+}+\mathrm{N}_{3}^{-}+\mathrm{K}+5 \mathrm{H}\right]^{+\cdot}$} \\
\hline $\mathbf{1}^{4+} \cdot 4 \mathrm{NO}_{3}^{-}$ & 418.9 & {$\left[\mathbf{1}^{4+}+2 \mathrm{NO}_{3}^{-}+2 \mathrm{~K}+5 \mathrm{H}\right]^{2+\bullet}$} & $2^{4+} \cdot 4 \mathrm{NO}_{3}^{-}$ & $\begin{array}{l}230.9 \\
361.7\end{array}$ & $\begin{array}{l}{\left[2^{4+}+\mathrm{NO}_{3}^{-}\right]^{3+}} \\
{\left[\left(2^{4+}\right)_{2}+3 \mathrm{NO}_{3}^{-}\right]^{4+\cdot}}\end{array}$ \\
\hline $\mathbf{1}^{4+} \cdot 4 \mathrm{HSO}_{4}^{-}$ & $\begin{array}{l}363.3 \\
519.6 \\
\end{array}$ & $\begin{array}{l}{\left[\mathbf{1}^{4+}+\mathrm{HSO}_{4}^{-}-\mathrm{H}\right]^{2+\cdot}} \\
{\left[\left(\mathbf{1}^{4+}\right)_{2}+3 \mathrm{HSO}_{4}^{-}+7 \mathrm{H}\right]^{3+\cdot}}\end{array}$ & $\mathbf{2}^{4+} \cdot 4 \mathrm{HSO}_{4}^{-}$ & 826.9 & {$\left[2^{4+}+\mathrm{HSO}_{4}^{-}+2 \mathrm{Ka}+\mathrm{Na}-\mathrm{H}\right]^{+\bullet}$} \\
\hline
\end{tabular}

表 $3\left[\mathbf{1}^{4+} \cdot 2 \mathrm{PF}_{6}^{-} \cdot 2 \mathrm{NO}_{3}^{-} \cdot 10 \mathrm{H}_{2} \mathrm{O}\right],\left[\mathbf{1}^{4+} \cdot 4 \mathrm{HSO}_{4}^{-} \cdot \mathrm{H}_{2} \mathrm{O}\right]$ 和 $\left[\mathbf{2}^{4+} \cdot 2 \mathrm{PF}_{6}^{-} \cdot 2 \mathrm{H}_{2} \mathrm{PO}_{4}^{-} \cdot 9.5 \mathrm{H}_{2} \mathrm{O}\right]$ 的晶体数据小结

Table 3 X-ray crystallographic data for $\left[\mathbf{1}^{4+} \cdot 2 \mathrm{PF}_{6}^{-} \cdot 2 \mathrm{NO}_{3}^{-} \cdot 10 \mathrm{H}_{2} \mathrm{O}\right],\left[\mathbf{1}^{4+} \cdot 4 \mathrm{HSO}_{4}^{-} \cdot \mathrm{H}_{2} \mathrm{O}\right]$ and $\left[\mathbf{2}^{4+} \cdot 2 \mathrm{PF}_{6}^{-} \cdot 2 \mathrm{H}_{2} \mathrm{PO}_{4}^{-} \cdot 9.5 \mathrm{H}_{2} \mathrm{O}\right]$

\begin{tabular}{llll}
\hline Compd. & {$\left[\mathbf{1}^{4+} \cdot 2 \mathrm{PF}_{6}^{-} \cdot 2 \mathrm{NO}_{3}^{-} \cdot 10 \mathrm{H}_{2} \mathrm{O}\right]$} & $\mathbf{1}^{4+} \cdot 4 \mathrm{HSO}_{4}^{-} \cdot \mathrm{H}_{2} \mathrm{O}$ & $\mathbf{2}^{4+} \cdot 2 \mathrm{PF}_{6}^{-} \cdot 2 \mathrm{H}_{2} \mathrm{PO}_{4}^{-} \cdot 9.5 \mathrm{H}_{2} \mathrm{O}$ \\
\hline CCDC No. & 979250 & 979249 & 842434 \\
Description & Prism & Prism & Block \\
Color & Colorless & Colorless & Colorless \\
From solution & Water/DMF/acetonitrile & Water/DMF/acetonitrile & Water/acetonitrile \\
Empirical formula & $\mathrm{C}_{38} \mathrm{H}_{54} \mathrm{~F}_{12} \mathrm{~N}_{12} \mathrm{O}_{15} \mathrm{P}_{2}$ & $\mathrm{C}_{40} \mathrm{H}_{41} \mathrm{~N}_{11} \mathrm{O}_{17} \mathrm{~S}_{4}$ & $\mathrm{C}_{38} \mathrm{H}_{38} \mathrm{~F}_{12} \mathrm{~N}_{10} \mathrm{O}_{17.50} \mathrm{P}_{4}$ \\
$M_{\mathrm{r}}$ & 1224.87 & 1078.09 & 1266.66 \\
Crystal size/mm & $0.21 \times 0.11 \times 0.09$ & $0.17 \times 0.10 \times 0.06$ & $0.34 \times 0.28 \times 0.16$ \\
Crystal system & Triclinic & Monoclinic & Orthorhombic \\
Space group & $P-1$ & $P 2(1) / n$ & $P 2(1) 2(1) 2(1)$ \\
$a / \AA$ & $8.9512(18)$ & $13.467(3)$ & $12.125(2)$ \\
$b / \AA$ & $10.749(2)$ & $12.395(3)$ & $16.355(3)$ \\
$c / \AA$ & $12.896(3)$ & $15.649(3)$ & $27.163(5)$ \\
$\alpha /\left({ }^{\circ}\right)$ & $91.96(3)$ & 90.00 & 90.00 \\
$\beta /\left({ }^{\circ}\right)$ & $92.38(3)$ & $95.73(3)$ & 90.00 \\
$\gamma /\left({ }^{\circ}\right)$ & $93.96(3)$ & 90.00 & 90.00 \\
$V / \AA^{3}$ & $1235.9(4)$ & $2599.1(9)$ & $5386.3(19)$ \\
$d /\left(\mathrm{g} \cdot \mathrm{cm}^{-3}\right)$ & 1.646 & 1.378 & 1.562 \\
$Z$ & 1 & 2 & 4 \\
$T / \mathrm{K}$ & $223(2)$ & $223(2)$ & $223(2)$ \\
$R_{1}, w R_{2}[I>2 \sigma(I)]$ & $0.1136,0.2553$ & $0.0876,0.2308$ & $0.0959,0.2042$ \\
$R_{1}, w R_{2}($ all data $)$ & $0.1614,0.2801$ & $0.1455,0.2594$ & $0.1260,0.2243$ \\
$Q$ Quality of fit & 1.005 & 1.028 & 0.961 \\
\hline
\end{tabular}




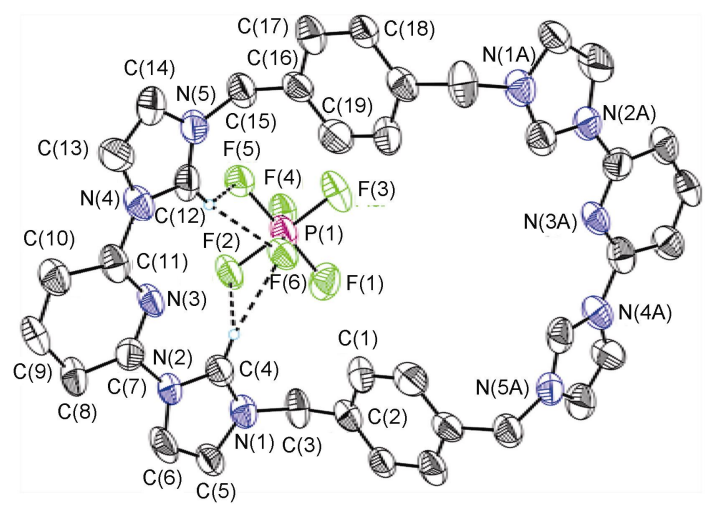

图 $6\left[\mathbf{1}^{4+} \cdot 2 \mathrm{PF}_{6}^{-} \cdot 2 \mathrm{NO}_{3}^{-} \cdot 10 \mathrm{H}_{2} \mathrm{O}\right]$ 的单晶结构图

Figure 6 Single crystal X-ray structure of $\left[\mathbf{1}^{4+} \cdot 2 \mathrm{PF}_{6}^{-} \cdot 2 \mathrm{NO}_{3}^{-}\right.$. $\left.10 \mathrm{H}_{2} \mathrm{O}\right]$

Selected interatomic distances of intermolecular hydrogen bonds $[\AA]$ : $\mathrm{F}(2) \cdots \mathrm{C}(4) \quad 2.515(6), \quad \mathrm{F}(5) \cdots \mathrm{C}(12) \quad 2.349(7), \quad \mathrm{F}(6) \cdots \mathrm{C}(12)$ 2.281(7), $\mathrm{F}(6) \cdots \mathrm{C}(4)$ 2.246(8). All the other anions and solvent molecules have been omitted for clarity

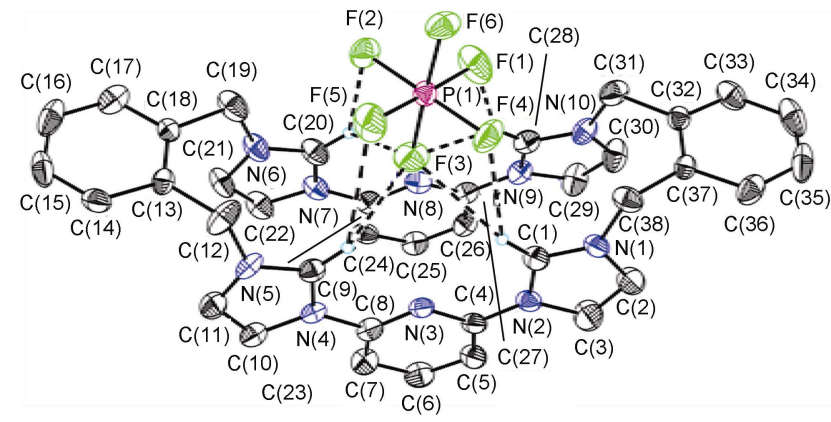

图 $7\left[2^{4+} \cdot 2 \mathrm{PF}_{6}^{-} \cdot 2 \mathrm{H}_{2} \mathrm{PO}_{4}^{-} \cdot 9.5 \mathrm{H}_{2} \mathrm{O}\right]$ 的单晶结构图

Figure 7 Single crystal X-ray structure of $\left[2^{4+} \cdot 4 \mathrm{PF}_{6}^{-}\right.$. $\left.2 \mathrm{H}_{2} \mathrm{PO}_{4}^{-} \cdot 9.5 \mathrm{H}_{2} \mathrm{O}\right]$

Selected interatomic distances of intermolecular hydrogen bonds $[\AA]$ : $\mathrm{F}(1) \cdots \mathrm{C}(28) 2.294, \mathrm{~F}(2) \cdots \mathrm{C}(20) 2.672, \mathrm{~F}(3) \cdots \mathrm{C}(9) 2.314, \mathrm{~F}(4) \cdots \mathrm{C}(1)$ 2.645. All the other anions and solvent molecules have been omitted for clarity

而同一阴离子客体能够通过 $\mathbf{1}^{4+}$ 的部分 $\mathrm{C}-\mathrm{H}$ 键发 生分子间氢键作用, 也能通过 anion- $\pi$ 相互作用与之进 行结合的证据还来自于 $\left[\mathbf{1}^{4+} \cdot 4 \mathrm{HSO}_{4}^{-} \cdot \mathrm{H}_{2} \mathrm{O}\right]$ 晶体. 如图 8, 图 9 所示, 含 $\mathrm{S}(2)$ 的 $\mathrm{HSO}_{4}^{-}$与 $\mathbf{1}^{4+}$ 的结合主要通过分子间 氢键进行, $\mathrm{HSO}_{4}^{-}$上的 $\mathrm{O}(6)$ 到 $\mathrm{C}(13)$ 的距离小于 3.1(图 8 ); 含 $\mathrm{S}(1)$ 的 $\mathrm{HSO}_{4}^{-}$与 $\mathbf{1}^{4+}$ 则主要通过 anion- $\pi$ 相互作用 结合, 表现为 $\mathrm{HSO}_{4}^{-}$上的 $\mathrm{O}(2)$ 到 $\mathrm{C}(7), \mathrm{O}(2)$ 到 $\mathrm{N}(2)$ 的距 离小于 3.7 (图 9). 以上单晶结构的分析结果再次验证了 溶液研究得出的结论, 即 $\mathbf{1}^{4+}$ 由于它的空腔较大且有着 立体的空腔, 所以 $\mathrm{C}-\mathrm{H}$ 键难以全部参与到对阴离子的 协同作用中去，但是它更易于去与阴离子通过 anion- $\pi$ 的作用模式进行结合; 对于 $\mathbf{2}^{4+}$ 其在溶液中由于空腔更 小更易形成近于平面的结构, 4 个咪唑基团上酸性较大 的 $\mathrm{C}-\mathrm{H}$ 易于发挥协同作用共同结合阴离子.
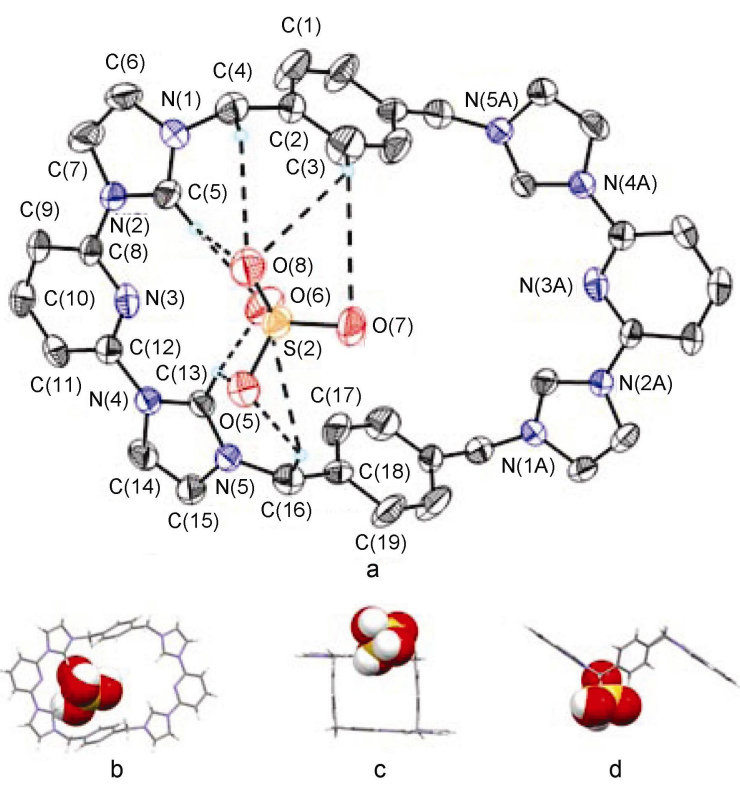

图 $8\left[\mathbf{1}^{4+} \cdot 4 \mathrm{HSO}_{4}^{-} \cdot \mathrm{H}_{2} \mathrm{O}\right]$ 的单晶结构图 1

Figure 8 Single crystal X-ray structures of $\left[\mathbf{1}^{4+} \cdot 4 \mathrm{HSO}_{4}^{-} \cdot \mathrm{H}_{2} \mathrm{O}\right]$ (1)

(a) Top view showing the ellipsoid form, (b) top, (c) side and (d) front views showing the stick form of the "partial chair" conformation of $\mathbf{1}^{4+}$ with a co-bound $\mathrm{HSO}_{4}^{-}$anion containing $\mathrm{S}(2)$ with framework form present in $\mathbf{1}^{4+} \cdot 4 \mathrm{HSO}_{4}^{-} \cdot \mathrm{H}_{2} \mathrm{O}$. Selected interatomic distances of intermolecular hydrogen bonds $[\AA]$ : $\mathrm{O}(5) \cdots \mathrm{C}(16) 3.036(5), \mathrm{O}(6) \cdots \mathrm{C}(5)$ 2.183(5), O(6) $\cdots \mathrm{C}(13)$ 2.113(5), O(7) $\cdots \mathrm{C}(3)$ 3.232(5), O(8) $\cdots \mathrm{C}(4)$ 2.734(4), $\mathrm{O}(8) \cdots \mathrm{C}(5) 3.032(5)$. All the other anions and solvent molecules have been omitted for clarity.

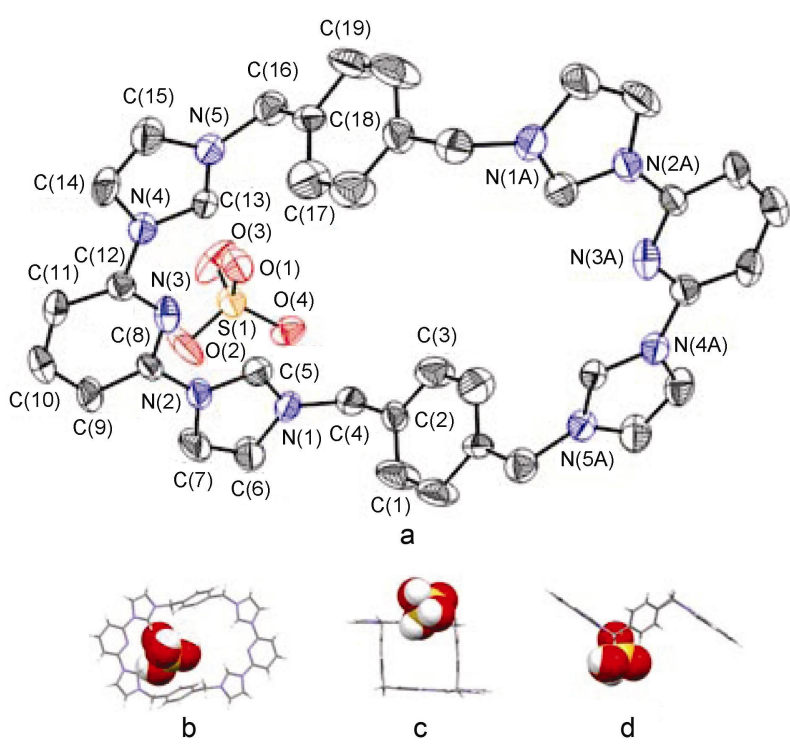

图 $9\left[1^{4+} \cdot 4 \mathrm{HSO}_{4}^{-} \cdot \mathrm{H}_{2} \mathrm{O}\right]$ 的单晶结构图 2

Figure 9 Single crystal X-ray structures of $\left[\mathbf{1}^{4+} \cdot 4 \mathrm{HSO}_{4}^{-} \cdot \mathrm{H}_{2} \mathrm{O}\right]$ (2)

(a) Top view showing the ellipsoid form, (b) top, (c) side and (d) front views showing the stick form of the "partial chair" conformation of $\mathbf{1}^{4+}$ with a co-bound $\mathrm{HSO}_{4}^{-}$anion containing $\mathrm{S}(1)$ with framework form present in $\mathbf{1}^{4+} \cdot 4 \mathrm{HSO}_{4}^{-} \cdot \mathrm{H}_{2} \mathrm{O}$. Possible anion $\cdots \pi$ interaction: Selected interatomic distances $[\AA]$ : $\mathrm{O}(2) \cdots \mathrm{N}(2) 3.508, \mathrm{O}(2) \cdots \mathrm{C}(7) 3.380, \mathrm{O}(3) \cdots$ $\mathrm{C}(5) 3.233, \mathrm{O}(3) \cdots \mathrm{N}(1) 3.610, \mathrm{O}(4) \cdots \mathrm{C}(4) 3.394, \mathrm{~S}(1) \cdots \mathrm{N}(1)$ 3.740. All the other anions and solvent molecules have been omitted for clarity 


\section{2 结论}

以 $d_{6}$-DMSO 为溶剂进行溶液当中的研究, 考察了 两个大环主体化合物 $\mathbf{1}^{4+}$ 或 $\mathbf{2}^{4+}$ 与一系列阴离子客体 $(\mathrm{Cl}$ ${ }^{-}, \mathrm{Br}^{-}, \mathrm{I}^{-}, \mathrm{N}_{3}^{-}, \mathrm{NO}_{3}^{-}$或 $\mathrm{HSO}_{4}^{-}$) 的相互作用. $\mathbf{1}^{4+}$ 与 $\mathbf{2}^{4+}$ 结构上的差异主要体现在它们的骨架上, 不同的桥连基 团使得 $\mathbf{1}^{4+}$ 比 $\mathbf{2}^{4+}$ 具有更为开放的立体空腔. 研究结果表 明: (1)与 $\mathbf{2}^{4+}$ 相比, 大环主体 $\mathbf{1}^{4+}$ 能够结合等量或者更多 的阴离子客体; (2)结合常数的计算表明, 即便主客体相 互作用具有不同平衡时的结合常数是难以比较的, 但是 如果只比较主客体形成 $1: 1$ 的复合物结合常数, $2^{4+}$ 与 阴离子的结合常数总是大于甚至是远大于 $1^{4+}$ 与强氢键 受体阴离子的结合常数; 但是对于较难形成分子间氢键 的阴离子如 $\mathrm{Br}^{-}$或 $\mathrm{I}^{-}$, 由于 anion- $\pi$ 作用的参与, 使得 $1^{4+}$ 与它们的结合常数约等于或大于 $2^{4+}$ 与它们的结合常 数. 推测产生上述现象的原因可能是由于 $\mathbf{2}^{4+}$ 上酸性较 强的 $\mathrm{C}-\mathrm{H}$ 键作用位点能协同与阴离子客体形成较强的 分子间氢键, 主要是通过 $\mathrm{C}-\mathrm{H}$ 键与阴离子进行结合; 而 $\mathbf{1}^{4+}$ 有着立体的空腔, 具有强酸性的咪唑基团 2 位 $\mathrm{C}-\mathrm{H}$ 键难以全部参与对阴离子的相互作用中去, 但更 易于同时与阴离子通过 anion- $\pi$ 的作用模式进行结合, 如 $\mathrm{Br}^{-}$或 $\mathrm{I}^{-}$. 单晶结构的研究也验证了溶液研究的结论. 上述结果涵盖具有各种形貌的无机阴离子, 具有普遍意 义.

\section{3 实验部分}

\section{1 仪器与试剂}

${ }^{1} \mathrm{H}$ NMR 的测定均通过 Bruker Avance $600 \mathrm{MHz}$ 核 磁共振波谱仪完成; 气相中质谱的测定均通过 SHIMADZU 公司 LCMS-2010 型液质联用仪完成; 单晶 结构的测定通过 Rigaku 单晶衍射仪 ST Saturn724+进 行.

实验过程所用溶剂均为分析纯. 溶剂及化学试剂均 购买于百灵威公司. 氝代二甲亚砜 $\left(d_{6}-\mathrm{DMSO}\right)$ 为进口试 剂(CIL).

主客体相互作用时涉及的阴离子种类包括 $\mathrm{Cl}^{-}$, $\mathrm{Br}^{-}, \mathrm{I}^{-}, \mathrm{N}_{3}^{-}, \mathrm{NO}_{3}^{-} \mathrm{HSO}_{4}^{-}$, 在工作中均以它们的四正 丁基铵盐(tetrabutylammonium; $\mathrm{TBA}^{+}$. 即 $\mathrm{TBA}^{+} \cdot \mathrm{Cl}^{-}$, $\mathrm{TBA}^{+} \cdot \mathrm{Br}^{-}, \mathrm{TBA}^{+} \cdot \mathrm{I}^{-}, \mathrm{TBA}^{+} \cdot \mathrm{NO}_{3}^{-}, \mathrm{TBA}^{+} \cdot \mathrm{HSO}_{4}^{-}$, $\mathrm{TBA}^{+} \cdot \mathrm{N}_{3}^{-}$)开展研究.

$\mathbf{1}^{4+}$ 和 $\mathbf{2}^{4+}$ 的六氟磷酸盐(即 $\mathbf{1}^{4+} \cdot 4 \mathrm{PF}_{6}^{-}$或 $\mathbf{2}^{4+} \cdot 4 \mathrm{PF}_{6}^{-}$) 参照文献 $[35,36]$ 的方法合成并纯化.

\section{2 实验方法}

\subsection{1 复合物的 Job's plot 测定}

采用 Job's plot 测定主客体分子之间的复合计量比
时. 保持主客体的总浓度为一恒定值(具体浓度值见结 果与讨论部分)进行 Job's plot 的测定 ${ }^{[39,40]}$.

\subsection{2 主客体复合物结合常数的测定方法}

结合常数用来表征主客体间形成复合物的稳定性. 本文采用 ${ }^{1} \mathrm{H}$ NMR 滴定法测定结合常数 ${ }^{[39,41]}$.

\section{2 .3 单晶培养}

通过单晶结构的测定可以获得主客体在固相当中 相互作用的具体信息. 本研究中所涉及的单晶样品采用 缓慢挥发法获得. 把 $1: 5$ 的大环主体化合物和客体阴 离子对应的四正丁基铵盐溶解在混合溶剂 $\mathrm{N}, \mathrm{N}$-二甲基 甲酰胺(DMF)和 $\mathrm{H}_{2} \mathrm{O}$ 中 $(V: V=1: 1)$. 静置, 室温下缓 慢挥发.

\subsection{4 质谱样品的制备}

通过质谱的测定可以获得主客体在气相当中相互 作用的相关信息. 本研究中所涉及的质谱样品采用离子 交换法获得. 把 $1: 5$ 的大环主体化合物和客体阴离子 对应的四正丁基铵盐溶解在乙腈中搅拌待出沉淀后过 滤，然后用乙腈冲洗过滤所得产物即获得质谱样品.

辅助材料(Supporting Information) 本文所涉及的晶 体结构数据 $\left(\left[\mathbf{1}^{4+} \cdot 2 \mathrm{PF}_{6}^{-} \cdot 2 \mathrm{NO}_{3}^{-} \cdot 10 \mathrm{H}_{2} \mathrm{O}\right](\mathrm{CCDC}\right.$ No. 979250), $\left[\mathbf{1}^{4+} \cdot 4 \mathrm{HSO}_{4}^{-} \cdot \mathrm{H}_{2} \mathrm{O}\right](\mathrm{CCDC}$ No. 979249$)$ )的 cif 及 checkcif 文件. 这些材料可以免费从本刊网站(http:// sioc-journal.cn/)上下载.

\section{References}

[1] Lehn, J. M.; Atwood, J. L.; Davies, L. E. Comprehensive Supramolecular Chemistry, Pergamon, New York, 1996, p. 27.

[2] Shen, J.-C. Supramolecular Layered Structures-Assembly and Functionalization, Science Press, Beijing, 2004, p. 1 (in Chinese). (沈家骢, 超分子层状结构——组装与功能, 科学出版社, 北京, 2004, p. 1.)

[3] Liu, Y.; You, C.-C.; Zhang, H.-Y. Supramolecular Chemistry-Molecular Recognition and Assembly of Synthesized Receptors, Nankai University Press, Tianjin, 2001, p. 1 (in Chinese). (刘育, 尤长城, 张衡益, 超分子化学—合成受体的分子识别 与组装, 南开大学出版社, 天津, 2001, p. 1.)

[4] Lehn, J. M. Angew. Chem., Int. Ed. 1988, 27.

[5] Dodziuk, H. Introduction to Supramolecular Chemistry, Kluwer Academic Publishers, London, 2002.

[6] Lehn, J. M. Proc. Natl. Acad. Sci. 2002, 99, 4763.

[7] Steed, J. W.; Atwood, J. L. Supramol. Chem. 2006, 62.

[8] Levason, W.; Reid, G.; Ncleverty, J. A. Comprehensive Coordination Chemistry II, Pergamon, Oxford, 2004, p. 18.

[9] Prauzsch, V.; Ibach, S.; Vogtle, F. J. Inclusion Phenom. Macrocylic Chem. 1993, 33, 427.

[10] Gale, P. A. Chem. Commun. 2011, 47, 82.

[11] Sessler, J. L.; Gale, P. A.; Stoddart, J. F.; Cho, W. S. Anion Receptor Chemistry; Monographs in Supramolecular Chemistry, Royal Society of Chemistry, Cambridge, U. K., 2006.

[12] Wenzel, M.; Hiscock, J. R.; Gale, P. A. Chem. Soc. Rev. 2012, 41, 480 . 
[13] (a) Beer, P. D.; Gale, P. A. Angew. Chem., Int. Ed. 2001, 40, 486. (b) Gong, H.-Y.; Xu, L.-J.; Zhou, L. Acta Chim. Sinica 2014, 72, 447 (in Chinese).

(龚汉元, 徐立进, 周丽, 化学学报, 2014, 72, 447.)

[14] (a) Ryo, S.; Li, W.; Kakuchi, T. Macromolecules 2011, 44, 4249.

(b) Zhang, D.-S.; Chen, J.-P.; Zeng, Y.; Yu, T.-J.; Li, Y. Chin. J. Org. Chem. 2013, 33, 110 (in Chinese).

(张读山, 陈金平, 曾毅, 于天君, 李嫕, 有机化学, 2013, 33, 110.)

[15] Lehn, J. M.; Hosseini, M. W.; Sessions, R. B. J. Am. Chem. Soc. 1981, 103, 1282.

[16] Lehn, J. M.; Hosseini, M. W. J. Am. Chem. Soc. 1987, 109, 7047.

[17] (a) Liu, G.; Shao, J. Acta Chim. Sinica 2011, 69, 1070 (in Chinese). (刘阁, 邵杰, 化学学报, 2011, 69, 1070.)

(b) Li, Y.; Zhang, F.; Zou, L.-B.; Bao, X.-P. Chin. J. Org. Chem. 2013, 33, 2485 (in Chinese).

(刘勇, 张峰, 邹林波, 蹇军友, 鲍小平, 有机化学, 2013, 33, 2485.)

[18] Sessler, J. L.; Tomat, E. Acc. Chem. Res. 2007, 40, 371.

[19] Sessler, J. L.; Park, J. S.; Karnas, E.; Ohkubo, K. Science 2010, 329, 1324.

[20] Alcalde, E.; Dinares, I.; Mesquida, N. Top. Heterocycl. Chem. 2010, $24,267$.

[21] Willans, C. E.; Anderson, K. M.; Potts, L. C.; Steed, J. W. Org. Biomol. Chem. 2009, 7, 1500.

[22] Jiang, L.-S.; Yang, D.-K. Acta Chim. Sinica 2012, 70, 1385 (in Chinese).

(蒋腊生, 杨登科, 化学学报, 2012, 70, 1385.)

[23] Yoon, J.; Xu, Z.; Singh, N. J.; Sook, K.; Spring, D. R.; Kim, K. S. Chem. Eur. J. 2011, 17, 1163.
[24] Yoon, J.; Xu, Z.; Kim, S. K. Chem. Soc. Rev. 2010, 39, 1457.

[25] Yoon, J.; Kim, J. K.; Singh, N. J.; Kim, K. S. Chem. Soc. Rev. 2006, $35,355$.

[26] Ihm, H.; Yun, S.; Kim, H. G.; Kim, J. K.; Kim, K. S. Org. Lett. 2002, 4, 2897.

[27] Kim, S. K. Angew. Chem., Int. Ed. 2005, 44, 2899.

[28] Gao, G.; You, J.-S.; Zhou, H.-J. Chem. Commun. 2013, 49, 1832.

[29] You, J.-S.; Zhou, H.-J.; Gao, G. J. Am. Chem. Soc. 2013, 135 , 14908.

[30] Hua, Y.; Flood, A. H. Chem. Soc. Rev. 2010, 39, 1262.

[31] Wei, T.-B.; Lin, Q.; Zhang, Y.-M. Sci. China, Ser. B 2004, 47, 295 (in Chinese).

(魏太保, 林奇, 张有明, 中国科学(B 辑), 2004, 47, 295.)

[32] Sessler, J. L.; Cai, J. J.; Gong, H. Y.; Arambula, J. F., Hay, B. P. J. Am. Chem. Soc. 2010, 132, 14058.

[33] Wei, T.-b.; Zhang, Y.-M. Chin. J. Org. Chem. 2009, 29, 575 (in Chinese). (魏太保, 张有明, 有机化学, 2009, 29, 575.)

[34] Wang, M.-X. Wang, D.-X. J. Am. Chem. Soc. 2013, 135, 892.

[35] Sessler, J. L.; Gong, H.-Y.; Rambo, B. M. Nat. Chem. 2010, $2,406$.

[36] Sessler, J. L.; Gong, H.-Y.; Rambo, B. M. J. Am. Chem. Soc. 2013, 135,6330 .

[37] Sessler, J. L.; Gong, H.-Y.; Rambo, B. M. J. Am. Chem. Soc. 2011, $133,1526$.

[38] Sessler, J. L.; Gong, H.-Y.; Rambo, B. M. Chem. Commun. 2011, $47,5973$.

[39] Oki, O. M. Applications of Dynamic NMR Spectroscopy to Organic Chemistry, VCH, Weinheim, 1985.

[40] Job, P. Ann. Chim. (Paris) 1928, 9, 113.

[41] Gans, P.; Sabatini, A.; Vacca, A. Talanta 1996, 43, 1739.

(Li, L.; Fan, Y.) 